\begin{tabular}{|c|l|}
\hline Title & The covalent modification of spectrin in red cell membranes by the lipid peroxidation product 4-hydroxy-2-nonenal \\
\hline Author(s) & A rashiki, Nobuto; Otsuka, Y ay oi; Ito, Daisuke; Y ang, Mira; Komatsu, Tomohiko; Sato, Kota; Inaba, Mutsumi \\
\hline Citation & $\begin{array}{l}\text { Biochemical and Biophysical Research Communications, 391(3), 1543-1547 } \\
\text { https://doi.org/10.1016/.bbrc.2009.12.121 }\end{array}$ \\
\hline Issue Date & 2010-01-15 \\
\hline Doc URL & http://hdl.handle.net/2115/43766 \\
\hline Type & article(author version) \\
\hline File Information & BBRC391-3_1543 1547.pdf \\
\hline
\end{tabular}

Instructions for use 


\title{
The covalent modification of spectrin in red cell membranes by the lipid peroxidation product 4-hydroxy-2-nonenal
}

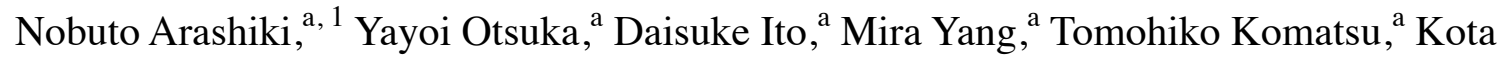 \\ Sato, and Mutsumi Inaba ${ }^{\mathrm{a}, *}$ \\ ${ }^{\mathrm{a}}$ Laboratory of Molecular Medicine, Graduate School of Veterinary Medicine, Hokkaido \\ University, Sapporo 060-0818, Japan \\ *Corresponding author: Mutsumi Inaba, Laboratory of Molecular Medicine, Department of \\ Veterinary Clinical Sciences, Graduate School of Veterinary Medicine, Hokkaido University, \\ Sapporo 060-0818, Japan, E-mail: inazo@vetmed.hokudai.ac.jp, Phone: +81-11-706-5580, \\ Fax: +81-11-706-5276 \\ ${ }^{1}$ Present address: Department of Biochemistry, Tokyo Women's Medical University School of \\ Medicine, Tokyo 162-8666, Japan
}

\section{ABSTRACT}

Spectrin strengthens the red cell membrane through its direct association with membrane lipids and through protein-protein interactions. Spectrin loss reduces the membrane stability and results in various types of hereditary spherocytosis. However, less is known about acquired spectrin damage. Here, we showed that $\alpha$ - and $\beta$-spectrin in human red cells are the primary targets of the lipid peroxidation product 4-hydroxy-2-nonenal (HNE) by immunoblotting and mass spectrometry analyses. The level of HNE adducts in spectrin (particularly $\alpha$-spectrin) and several other membrane proteins was increased following the HNE treatment of red cell membrane ghosts prepared in the absence of MgATP. In contrast, ghost preparation in the presence of MgATP reduced HNE adduct formation, with preferential $\beta$-spectrin modification and increased cross-linking of the HNE-modified spectrins. Exposure of intact red cells to HNE resulted in selective HNE-spectrin adduct formation with a similar preponderance of HNE- $\beta$-spectrin modifications. These findings indicate that HNE adduction occurs preferentially in spectrin at the interface between the skeletal proteins and lipid bilayer in red cells and suggest that HNE-spectrin adduct aggregation results in the extrusion of damaged spectrin and membrane lipids under physiological and disease conditions.

Key words: spectrin, 4-hydroxy-2-nonenal, lipid peroxidation, red cell, membrane

\section{Introduction}

Spectrin, a major constituent of the human red blood cell (RBC) membrane skeleton, acts as a scaffolding protein and mediates the organization and assembly of a diverse set of proteins in specialized membrane domains [1]. Spectrin is a flexible, rod-like protein formed 
by two end-to-end associated heterodimers composed of $\alpha$ and $\beta$ subunits, with sizes of 280 $\mathrm{kDa}$ and $246 \mathrm{kDa}$, respectively. In RBCs, the spectrin-actin network is linked to the lipid bilayer through protein-protein interactions, specifically spectrin-protein 4.1R-glycophorin $\mathrm{C}$ and spectrin-ankyrin-band 3 associations, and through direct association of spectrin with phosphatidylserine (PS) in the inner bilayer leaflet [2-4]. Such vertical interactions between the spectrin-actin skeleton and lipid bilayer play pivotal roles in maintaining RBC membrane stability $[3,5,6]$. Spectrin loss reduces the membrane mechanical resilience, leading to spherocyte formation in various types of hereditary spherocytosis [4, 7]. In contrast with its role in congenital disorders, less is known about acquired spectrin damage. Previous studies have focused on the oxidation of spectrin sulfhydryl groups, revealing that RBC exposure to oxidative conditions causes morphological and mechanistic changes, principally through spectrin oxidation $[8,9]$.

In contrast, membrane phospholipid peroxidation generates various cytotoxic aldehydes that can damage proteins and other cellular constituents [10-12]. 4-Hydroxy-2-nonenal (HNE) is the most abundant and toxic aldehyde generated by the oxidation of plasma membrane polyunsaturated fatty acids, such as arachidonic acid. HNE is a highly reactive electrophile that reacts with the side chains of various amino acid residues, including Cys, His, and Lys, to form Michael adducts [10, 13]. Protein modification by HNE causes serious detrimental effects in the cell, due to the resulting functional defects and cross-linking of proteins [14-18]. HNE also accumulates in RBCs [19], leading to the covalent modification of membrane proteins [20]. However, the target proteins for HNE modification remain unknown.

These findings have suggested that oxidative environments under physiological or disease conditions may cause spectrin modifications by HNE, leading to deleterious changes in the plasma membrane mechanical properties. Therefore, here we examined the presence and significance of covalent spectrin modifications by HNE in human RBCs. We used anti-HNE antibodies and matrix-assisted laser desorption/ionization time-of-flight mass spectrometry (MALDI-TOF MS) to identify HNE-modified proteins in RBC membranes.

\section{Materials and methods}

Preparation of RBC membrane ghosts and spectrin-actin extracts. After obtaining informed consent, blood was obtained from healthy volunteers. Preparation of RBC membrane ghosts and extraction of spectrin-actin were performed as described previously [6]. In some experiments, $\mathrm{RBC}$ ghosts were prepared by hemolysis in hypotonic buffer $(5 \mathrm{mM}$ Tris/ $\mathrm{Cl}[\mathrm{pH}$ 7.4], $5 \mathrm{mM} \mathrm{KCl}$, and $1 \mathrm{mM} \mathrm{MgCl} 2$ ) with or without $0.6 \mathrm{mM} \mathrm{MgATP}$ (Sigma), as described previously [3]. We also obtained ghosts from RBCs separated into different fractions using a discontinuous arabinogalactan gradient [21].

SDS-PAGE and immunoblotting. Membrane proteins were separated by SDS-PAGE on 6.5\% or $10 \%$ SDS-gels followed by staining with Coomassie brilliant blue or immunoblotting. Immunoblotting was performed as described previously [22] using antibodies against HNE-Michael adducts (Calbiochem), HNE-His (monoclonal antibody HNEJ-2; Nikken SEIL 
Co., Fukuroi, Japan), and spectrin [6].

MALDI-TOF MS analysis of spectrins. After separation by SDS-PAGE, proteins in the gel slices were reduced with $\mathrm{NaBH}_{4}$ for $30 \mathrm{~min}$ at an ambient temperature unless otherwise indicated. This was done to detect unstable reversibly formed adducts, such as HNE-Lys Michael adducts [23]. The proteins were subsequently stained with Coomassie brilliant blue and digested in gel slices with $10 \mu \mathrm{g} / \mathrm{ml}$ of trypsin Gold (Promega) for $16 \mathrm{~h}$ at $37^{\circ} \mathrm{C}$. Prior to protease digestion, the Cys residues were reduced with $10 \mathrm{mM}$ dithiothreitol and alkylated with $55 \mathrm{mM}$ iodoacetamide. Peptides were eluted from gels, desalted using C18 ZipTips (Millipore), and crystallized using saturated $\alpha$-cyano-4-hydroxy-cinnamic acid (Bruker Daltonics) as a matrix. Full-scan mass spectra of the tryptic peptides from 800-3,000 m/z were collected in positive mode by averaging 100-250 spectra using a Bruker autoflex MALDI-TOF mass spectrometer. Measured peptide masses were used to search the NCBI and Swiss-Prot sequence database for protein identification using MASCOT software.

In vitro formation of $H N E$-protein adducts in RBCs and RBC ghosts. A packed $10 \%$ cellular volume of RBCs was suspended in PBS containing $5 \mathrm{mM}$ glucose. After incubation for various times (0-6 h) at $37^{\circ} \mathrm{C}$ in the presence or absence of 0.1-1.0 $\mathrm{mM} \mathrm{HNE}$ (Calbiochem), the cells were washed with PBS. Membrane ghosts were then prepared as described above. Ghost proteins were labeled with HNE by incubating PBS-suspended ghosts ( $1 \mathrm{mg}$ protein $/ \mathrm{ml}$ ) with $0.01-1.0 \mathrm{mM} \mathrm{HNE}$ at $37^{\circ} \mathrm{C}$ for appropriate time periods $(0-60 \mathrm{~min})$, followed by SDS-PAGE and immunoblotting. In some experiments, incubation was performed in hypotonic buffer with $0.1 \mathrm{mM} \mathrm{MgATP}$.

Statistical analysis. Paired Student $t$ test was used to assess statistical significance.

\section{Results}

\section{HNE modification of $\alpha$ - and $\beta$-spectrin in human RBC membranes}

Immunoblotting analysis revealed the presence of polypeptides that had reacted with the anti-HNE antibody. These polypeptides were located at positions corresponding to $\alpha$ - and $\beta$-spectrin in the freshly prepared human RBC membranes and in the crude spectrin-actin preparation extracted from the ghosts (Fig. 1A). These signals were weak and required $>15$ sec for detection when 5-10 $\mu \mathrm{g}$ of membrane proteins were loaded. Spectrin, especially $\beta$-spectrin, also reacted with the anti-HNE-His adducts, suggestive of a predominance of HNE-His adducts in $\beta$-spectrin (Fig. 1A).

We then examined the contents of HNE-modified spectrin in density-separated RBCs to examine if these adducts accumulate during RBC aging. The lightest/youngest cell fraction showed HNE/spectrin ratios equivalent to those in fractions 3 and 4, which comprised $\sim 90 \%$ of total cell counts (Fig. 1B). No statistically significant differences in the HNE levels of $\alpha-$ and $\beta$-spectrin were observed among fractions 1 through 6 , although the densest fractions (fractions 5 and 6) had reduced $\mathrm{HNE} /$ spectrin ratios; an increase would have been expected if there was accumulation of HNE-spectrin adducts during RBC senescence. These data suggested that human RBCs possess HNE-modified spectrin throughout their life-span. 
We analyzed the positions where these spontaneous modification with HNE occurred in spectrin polypeptides from freshly prepared RBC ghosts. MALDI-TOF MS detected 50 70 tryptic peptides that covered $\sim 25-35 \%$ of the total amino acid residues of $\alpha$ - and $\beta$-spectrin. Several independent analyses consistently showed that a dozen or more distinct peptides derived from either $\alpha$ - and $\beta$-spectrin contained one or more Michael-type HNE-amino acid adduct and Schiff base conjugate (Fig. 2 and Supplementary Table 1). For example, in $\beta$-spectrin reduced with $\mathrm{NaBH}_{4}$, the MALDI-TOF MS consistently detected an

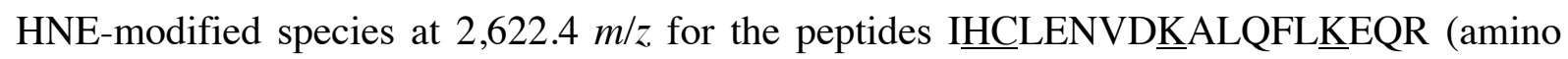
acid residues 110-127) derived from the $\mathrm{N}$-terminal actin-binding domain of this subunit. The observed mass shift of $438 \mathrm{~m} / \mathrm{z}(=158+2 \times 140 \mathrm{~m} / \mathrm{z})$ was indicative of a Michael addition reaction with mass shifts of $158 \mathrm{~m} / z$ at either the 2nd or 3rd underlined His or Cys residue, respectively. The mass shift of $438 \mathrm{~m} / \mathrm{z}$ was also indicative of Schiff base adducts with mass shifts of $280 \mathrm{~m} / \mathrm{z}(=2 \times 140 \mathrm{~m} / \mathrm{z})$ at the two underlined Lys residues. This is because reduction with $\mathrm{NaBH}_{4}$ increases the characteristic mass shifts of 156 and $138 \mathrm{~m} / z$ for Michael and Schiff base adducts, respectively, by 2 atomic mass units [23]. When $\beta$-spectrin was analyzed without reduction, peaks at $1,382.6$ and $2,239.2 \mathrm{~m} / \mathrm{z}$, shifted by $312(=2 \times 156)$ and $468(=3$ $\times 156)$ atomic mass units, respectively, were detected for the corresponding peptides IHCLENVDK and IHCLENVDKKALQFLK, suggesting that all underlined residues could form Michael-type HNE adducts. Together with the data for the mass shifts of several other peptides (Supplementary Table 1), these results allow us to predict that all Cys-, His-, and Lys-Michael adducts and Schiff base Lys adducts are present in both $\alpha$ - and $\beta$-spectrin, consistent with our immunoblot results with anti-HNE antibodies. Fig. 2 illustrates the positions and types of HNE adduct formation obtained by MALDI-TOF MS for $\alpha$ - and $\beta$-spectrin.

\section{Labeling of spectrins with extrinsic $\mathrm{HNE}$}

We next examined the covalent modification of spectrin in RBCs incubated with extrinsic HNE. Membrane ghosts treated with $0.1 \mathrm{mM}$ HNE exhibited a marked increase in HNE-spectrin adduct contents within $10 \mathrm{~min}$ of incubation (Fig. 3A). Incubation also caused HNE labeling of several other membrane proteins with apparent sizes of 115, 110, 100, 80, and $43 \mathrm{kDa}$ (Fig. 3A). These polypeptides were likely $\alpha$ - and $\beta$-adducin, band 3 , protein $4.1 \mathrm{R}$, and actin, respectively, judging from their migrating positions and antibody reactivities (data not shown). In contrast, when intact RBCs were incubated with HNE for several hours, a remarkable increase in the signals of HNE-protein adducts was found for spectrin, especially $\beta$-spectrin, but not for other membrane proteins (Fig. 3B). These results demonstrated that HNE selectively reacted with spectrin in vivo and suggested that $\alpha$ - and $\beta$-spectrin had different status in intact RBCs and in the isolated membrane ghosts, so as to cause distinct patterns in HNE adduct formation.

\section{Effect of spectrin-lipid association on HNE modification of $\alpha$ - and $\beta$-spectrin}

Therefore, we next compared spectrin labeling with HNE in membrane ghosts possessing different association status of spectrin with membrane lipids, i. e., ghosts prepared 
in the presence (MgATP ghost) or absence (control ghost) of MgATP [3]. When ghosts were incubated with increasing HNE concentrations, we observed marked increases in the intensities of HNE-spectrin adducts in both MgATP and control ghosts (Fig. 4). In principle, when membrane proteins containing equivalent amounts of spectrins were exposed to HNE, spectrin labeling with HNE in the MgATP ghost was less abundant than in the control ghost, and was much less than that in the crude spectrin-actin extract (Fig. 4A).

Remarkable differences between the MgATP and control ghosts were observed at lower HNE concentrations (0.05-0.3 mM). Immunoblotting revealed that the abundance of HNE signals relative to spectrin contents was significantly higher in the control than in the MgATP ghost (Figs. 4B and 4C), demonstrating that HNE spectrin labeling preferentially occurred in the control ghosts. In contrast, the MgATP ghost showed reductions in the spectrin contents and corresponding increases in high molecular weight aggregate formation, with prominent signals for both the anti-HNE adducts and anti-spectrin antibodies (Fig. 4B). These data suggest that HNE-modified spectrins in the MgATP ghosts tended to be cross-linked and aggregated, although the adduction levels were lower than those in control ghosts. Moreover, the HNE labeling of $\beta$-spectrin was nearly equivalent to that of $\alpha$-spectrin in the MgATP ghost, whereas HNE labeling was predominantly found in $\alpha$-spectrin in the control ghost, as observed in the crude spectrin-actin extract (Figs. 4A-4C).

\section{Discussion}

Various diseases involve proteins modified by HNE and protein cross-linking through HNE adducts contributes to disease pathogenecities [14, 16-18]. Here, we showed that spectrins are the primary targets of $\mathrm{HNE}$, and that HNE-modified spectrins generate irreversibly cross-linked aggregates in human RBCs. Since HNE accumulates in RBCs in circulation [19], the extensive modification of spectrins by HNE may occur in peripheral blood RBCs under physiological and pathological conditions.

The relative abundance of HNE-spectrin adducts was higher in control ghosts than in MgATP ghosts. MgATP helps maintain the asymmetric distribution of PS through the action of MgATP-dependent phospholipid translocase. Interactions between aminophospholipids and skeletal proteins modulate the human RBC membrane mechanical stability [3]. The spectrin-actin network is thus tightly associated with the lipid bilayer through the direct binding of spectrin with PS in the presence of MgATP [3] as well as through protein-protein interactions [4]. Therefore, in the absence or under reduced concentrations of MgATP, greater HNE adduct formation may occur in spectrins that are dissociated from the lipid bilayer and have increased susceptibility to the amphiphilic HNE. Then, local aggregation of HNE-modified spectrins is inevitable, since these spectrins contain Cys-, His-, and Lys-Michael adducts and Schiff bases on the Lys residues, that are required for intra- or intermolecular cross-linking $[10,12,13,16,17]$. Local spectrin aggregation may free the lipid bilayer from underlying spectrin-actin skeletons, possibly leading to membrane surface area loss by extrusion, which occurs in RBCs under oxidative conditions [8,9] and various RBC membrane disorders [4, 7]. Thus, in addition to modulating RBC membrane mechanical 
properties, the spectrin-membrane lipid interactions may have an obligatory role in preventing skeletal proteins from excessive HNE adduct formation.

In contrast to the observations under ATP-free conditions described above, in which HNE modifications preferentially occurred in $\alpha$-spectrin (Figs. 3 and 4), the intensities of $\beta$-spectrin HNE adducts were comparable to those of $\alpha$-spectrin in HNE-exposed MgATP ghosts (Fig. 4). Furthermore, $\beta$-spectrin was the principal target of HNE when intact RBCs were exposed to HNE (Fig. 3). Spectrin reportedly associates directly with PS [2,3] in several distinct segments involving the $\mathrm{N}$-terminal actin-binding domain, triple helical structural repeats 2-4 and 12-14 of $\beta$-spectrin, and repeats 8-10 of $\alpha$-spectrin [24]. Thus, under physiological conditions, HNE adduction occurs preferentially in membrane-associated $\beta$-spectrin at the interface of the skeletal protein and the inner leaflet of the membrane lipid bilayer. Intrinsic HNE is generated in the lipid bilayer by fatty acid peroxidation; extrinsic HNE reaches the inner leaflet presumably by diffusion through the lipid bilayer [12]. These assumptions are consistent with the presence of HNE adducts in the N-terminal regions of $\beta$-spectrin, including repeats 2-4 (Fig. 2). Moreover, selective HNE-spectrin formation and protection of other membrane proteins from HNE addition probably involve HNE elimination by cellular glutathione. Glutathione readily reacts with HNE via Michael addition spontaneously or enzymatically to form glutathione-HNE conjugate and much of aqueous HNE in a cell is bound to glutathione [13].

Interestingly, in the presence of MgATP, the HNE-modified spectrins tended to be cross-linked and aggregated (Fig. 4). HNE reportedly modifies the three His residues of amyloid $\beta$ protein $(A \beta)$. These HNE-modified $A \beta$ molecules have an increased affinity for membrane lipids and possess a conformation similar to that of mature amyloid fibrils $[16,25]$. The observed enrichment of HNE-His adducts (Fig. 1A and Supplementary Table 1) suggests that the propensity of HNE-modified spectrin to form aggregates may involve mechanisms similar to those found for $\mathrm{A} \beta$. Thus, the closer to the physiological environment the RBC membrane is, the more readily membrane damage through spectrin aggregation can occur. Consequently, restricted membrane surface area loss would occur with much less severity compared to pathological conditions described above. This mechanism, involving preferential $\beta$-spectrin HNE-modification and aggregate formation, may provide significant protection against excessive lipid peroxidation product accumulation during RBC aging. The slightly decreased contents of HNE-spectrin adducts in the most dense population (Fig. 1C), which displayed reduced membrane surface area/volume ratios possibly represent the gradual accumulation followed by the loss of aggregated HNE-modified proteins and membrane lipids during RBC aging. This mechanism also gives an explanation for the difference between ghosts from intact RBCs (Fig. 1) and ghosts from HNE-exposed RBCs (Fig. 3) in HNE modification patterns of $\alpha$ - and $\beta$-spectrin. HNE modification and consequent aggregate formation would be expected for spectrin family members expressed at the cytoplasmic plasma membrane surface of most metazoan cells [1].

In conclusion, our study demonstrates that HNE-spectrin adducts spontaneously form in human RBCs. Our data also suggest that HNE-spectrin aggregate formation may be involved in the physiological and pathological destruction of RBCs. 


\section{Acknowledgments}

The authors thank Drs. Yuichi Takakuwa and Sumie Manno (Tokyo Women's Medical University) for discussions and technical support. This work was supported by Grants-in-aid for Scientific Research 19658116 and 19208027 from the Japan Society for Promotion of Science (to M. I.).

\section{References}

[1] V. Bennett, J. Healy, Organizing the fluid membrane bilayer: diseases linked to spectrin and ankyrin, Trends Mol. Med. 14 (2008) 28-36.

[2] A. M. Cohen, S.-C. Liu, L. H. Derick, et al., Ultrastructural studies of the interaction of spectrin with phosphatidylserine liposomes, Blood 68 (1986) 920-926.

[3] S. Manno, Y. Takakuwa, N. Mohandas, Identification of a functional role for lipid asymmetry in biological membranes: phosphatidylserine-skeletal protein interactions modulate membrane stability, Proc. Natl. Acad. Sci. USA. 99 (2002) 1943-1948.

[4] N. Mohandas, P. G. Gallagher. Red cell membrane: past, present, and future, Blood 112 (2008) 3939-3948.

[5] P. S. Low, B. M. Willardson, N. Mohandas, et al., Contribution of the band 3-ankyrin interaction to erythrocyte membrane stability, Blood 77 (1991) 1581-1586.

[6] M. Inaba, A. Yawata, I. Koshino, et al., Defective anion transport and marked spherocytosis with membrane instability caused by hereditary total deficiency of red cell band 3 in cattle due to a nonsense mutation, J. Clin. Invest. 97 (1996) 1804-1817.

[7] S. Perrotta, P. G. Gallagher, N. Mohandas, Hereditary spherocytosis, Lancet 372 (2008) $1411-1426$.

[8] L. M. Snyder, N. L. Fortier, J. Trainer, et al., Effect of hydrogen peroxide exposure on normal human erythrocyte deformability, morphology, surface characteristics, and spectrin-hemoglobin cross-linking, J. Clin. Invest. 76 (1998) 1971-1977.

[9] G. M. Wagner, D. T.-Y. Chiu, J. H. Qju, et al., Spectrin oxidation correlates with membrane vesiculation in stored RBCs, Blood 69 (1987) 1777-1781.

[10] H. Esterbauer, R. J. Schauer, H. Zollner, Chemistry and biochemistry of 4-hydroxynonenal, malondialdehyde and related aldehydes, Free Rad. Biol. Med. 11 (1991) 81-128.

[11] C. Schneider, K. A. Tallman, N. A. Porter, et al., Two distinct pathways of formation of 4-hydroxynonenal. Mechanisms of nonenzymatic transformation of the 9- and 13-hydroperoxides of linoleic acid to 4-hydroxyalkenals, J. Biol. Chem. 276 (2001) 20831-20838.

[12] K. Uchida, Review. 4-Hydroxy-2-nonenal: a product and mediator of oxidative stress, Prog. Lipid Res. 42 (2003) 318-343.

[13] D. R. Petersen, J. A. Doorn, Reactions of 4-hydroxynonenal with proteins and cellular targets, Free Rad. Biol. Med. 37 (2004) 937-945.

[14] T. J. Montine, V. Amarnath, M. E. Martin, et al., E-4-Hydroxy-2-nonenal is cytotoxic and 
cross-links cytoskeletal proteins in P19 neuroglial cultures, Am. J. Pathol. 148 (1996) 89-93

[15] C. M. Lauderback, J. M. Hackett, F. F. Huang, et al., The glial glutamate transporter, GLT-1, is oxidatively modified by 4-hydroxy-2-nonenal in the Alzheimer's disease brain: the role of A $31-42$, J. Neurosci.78 (2001) 413-416.

[16] I. V. J. Murray, L. Liu, H. Komatsu, et al., Membrane-mediated amyloidogenesis and the promotion of oxidative lipid damage by amyloid $\beta$ proteins, J. Biol. Chem. 282 (2007) 9335-0345.

[17] B. J. Stewart, J. A. Doorn, D. R. Petersen, Residue-specific adduction of tubulin by 4-hydroxynonenal and 4-oxononenal causes cross-linking and inhibits polymerization, Chem. Res. Toxicol. 20 (2007) 1111-1119.

[18] J. Kokubo, N. Nagatani, K. Hiroki, et al., Mechanism of destruction of microtubule structures by 4-hydroxy-2-nonenal, Cell Struct. Funct. 33 (2008) 51-59.

[19] K. Ando, M. Beppu, K. Kitagawa, Evidence for accumulation of lipid hydroperoxides during the aging of human red blood cells in the circulation, Biol. Pharmacol. Bull. 18 (1995) 659-663.

[20] K. Uchida, Y. Hasui, T. Osawa, Covalent attachment of 4-hydroxy-2-nonenal to erythrocyte proteins, J. Biochem. 122 (1997) 1246-1251.

[21] T. Tiffert, V. L. Lew, H. Ginsburg, et al., The hydration state of human red blood cells and their susceptibility to invasion by Plasmodium falciparum, Blood 105 (2005) 4853-4860.

[22] D. Ito, I. Koshino, N. Arashiki, et al., Ubiquitylation-independent ER-associated degradation of an AE1 mutant associated with dominant hereditary spherocytosis in cattle, J. Cell Sci. 119 (2006) 3602-3612.

[23] F. Fenaille, P. A. Guy, J. C. Tabet, Study of protein modified by 4-hysroxy-2-nonenal and other short chain aldehydes analyzed by electrospray ionization tandem mass spectrometry, J. Am. Soc. Mass Spectrom. 14 (2003) 215-226.

[24] X. An, X. Guo, H. Sum, et al., Phosphatidylserine binding sites in erythroid spectrin: location and implications for membrane stability, Biochemistry 43 (2004) 310-315.

[25] L. Liu, H. Komatsu, I. V. J. Murray, et al., Promotion of amyloid $\beta$ protein misfolding and fibrillogenesis by a lipid oxidation product, J. Mol. Biol. 377 (2008) 1236-1250.

\section{Legends for Figures}

Fig. 1. HNE-spectrin adducts in human RBC membranes. A. Ghost membrane proteins $(G, 8$ $\mu \mathrm{g} / \mathrm{lane})$ and the crude spectrin/actin extracted from ghosts $(S, 2-3 \mu \mathrm{g} / \mathrm{lane})$ were separated on SDS-PAGE gels. Protein components were analyzed by Coomassie brilliant blue staining $(C B B)$ and HNE-protein adduct formation was assessed by immunoblotting using anti-HNE $(H N E)$ or anti-HNE-His (HNE-His) adduct antibodies. In immunoblotting, the membranes were exposed to the films for $1 \mathrm{~min}$. The migrating positions of the major membrane proteins, including $\alpha$ - and $\beta$-spectrin ( $\alpha$-Sp and $\beta$-Sp, respectively), are indicated. B. Human RBCs were separated into fractions 1 to 6 by centrifugation on an arabinogalactan density gradient [21]. Fractions were examined for their HNE-spectrin and whole spectrin contents as described above $(8 \mu \mathrm{g} / \mathrm{lane}$, exposed for $1 \mathrm{~min})$. C. Contents of HNE-modified $\alpha$-spectrin (hatched columns) and $\beta$-spectrin (unhatched columns) shown in B were quantified by 
densitometric scanning, normalized by the corresponding spectrin, and shown as the relative abundance of the HNE-spectrin (HNE/Spectrin). Data represent a typical result from several independent experiments.

Fig. 2. Schematic illustration of HNE-amino acid adduct positions in $\alpha$ - and $\beta$-spectrin deduced from MALD-TOF MS analysis. Positions of HNE-amino acid adducts found in $\alpha-$ and $\beta$-spectrin by MALDI-TOF MS analysis (Supplementary Table 1) are summarized and schematically presented. Closed and open circles indicate Michael-type and Schiff base adducts, respectively, for spectrins reduced with $\mathrm{NaBH} 4$ prior to MS analysis. Closed triangles indicate Michael adducts deduced for spectrins without reduction. Illustrations for spectrin and phosphatidylserine-binding repeats are according to previous studies [4, 24]. Interactions between spectrin and membrane proteins through glycophorin C-protein 4.1-spectrin and band 3-ankyrin-spectrin linkages are also shown.

Fig. 3. HNE-spectrin adduct formation in RBC ghosts and intact RBCs exposed to HNE. A. Human RBC ghosts were suspended in PBS containing 0.1 mM HNE and placed on ice for 10 min then incubated at $37^{\circ} \mathrm{C}$ for the indicated periods and analyzed for HNE-protein adduct formation by immunoblotting ( $5 \mu \mathrm{g} / \mathrm{lane})$ with the anti-HNE antibody. B. Human RBCs were incubated at $37^{\circ} \mathrm{C}$ in the presence of 0.1 or $1.0 \mathrm{mM} \mathrm{HNE}$ for 0,1 or $4 \mathrm{~h}$. RBC membrane ghosts were prepared and analyzed by immunoblotting ( $5 \mu \mathrm{g} / \mathrm{lane})$. Immunoblotting membranes were exposed to films for 3-5 sec. Arrowheads indicate $\alpha$ - and $\beta$-spectrin $(\alpha-S p$ and $\beta$-Sp) and some major proteins in the RBC membranes, including $\alpha$ - and $\beta$-adducin $(\alpha$-Add and $\beta$-Add $)$, band 3, protein 4.1, actin, and hemoglobin $(H b)$.

Fig. 4. HNE-spectrin adduct formation in MgATP ghosts and control ghosts exposed to HNE. A. Human RBC ghosts were prepared in hypotonic buffer $(5 \mathrm{mM}$ Tris/Cl. $\mathrm{pH} 7.4,5 \mathrm{mM} \mathrm{KCl}$, $1 \mathrm{mM} \mathrm{MgCl} 2$ ) with or with out $0.6 \mathrm{mM} \mathrm{MgATP}$ (MgATP ghost and Control ghost, respectively) and were diluted in the same buffer to contain $0.1 \mathrm{mM} \mathrm{MgATP}$ when present. These ghosts were incubated at $37^{\circ} \mathrm{C}$ and $1 \mathrm{mg}$ protein $/ \mathrm{ml}$ for $1 \mathrm{~h}$ with $0,0.05,0.1,0.2,0.3$, $0.4,0.5$, or $1.0 \mathrm{mM}$ HNE. After rapid centrifugation, the membrane proteins ( $1 \mu \mathrm{g} / \mathrm{lane})$ were analyzed for HNE-protein adducts by immunoblotting. Crude extracts containing spectrin and actin $(S p / A c t$ ext, $0.3 \mathrm{mg}$ protein $/ \mathrm{ml})$ were also incubated with $\mathrm{HNE}$ and processed to detect HNE-protein adducts $(0.3 \mu \mathrm{g} / \mathrm{lane})$. B. Typical immunoblots similar to those shown in A, with longer exposure time for HNE-protein adducts $(H N E)$, and the corresponding immunoblots for spectrin (Spectrin). In A and B, $\alpha$ - and $\beta$-spectrin ( $\alpha$-Sp and $\beta$-Sp) and high molecular weight species $(H M W)$ are indicated. Asterisks mark the top of the separating gel. Immunoblot membranes were exposed to films for $3 \mathrm{sec}$ and $10 \mathrm{sec}$ in $A$ and $B$, respectively. C. Relative abundance of HNE-spectrin adducts for $\alpha$ - and $\beta$-spectrin (open and closed circles, respectively) were obtained as described in the legend for Fig. 1. Data are the means \pm S.D. (n $=3)$. ${ }^{*} P<0.01$. The third panel displays data for the crude extracts of spectrin/actin obtained from immunoblots shown in A, and the corresponding immunoblots for spectrin (Sp/Act ext, $\mathrm{n}$ $=2)$. 
Supplementary Table 1. Analysis of HNE-modified peptides derived from $\alpha$ - and $\beta$-spectrin by MALDI-TOF MS.

Tryptic peptides derived from $\alpha$ - and $\beta$-spectrin were analyzed for Michael-type (Michael) and Schiff base (Schiff) HNE adducts by MALDI-TOF MS. The amino acid residues, sequences, and theoretical and observed masses are shown for peptides deduced to contain HNE adduct(s) by at least two independent analyses (peptides 1-16 and 1-18 for $\alpha$ - and $\beta$-spectrin, respectively). Localization of each peptide in the spectrin repeats is also indicated.

\begin{tabular}{|c|c|c|c|c|c|c|c|c|}
\hline \multirow[t]{2}{*}{ Spectrin } & \multirow[t]{2}{*}{$\begin{array}{l}\text { Peptide } \\
\text { number }\end{array}$} & \multirow[t]{2}{*}{$\begin{array}{l}\text { Spectrin } \\
\text { repeats }\end{array}$} & \multirow[t]{2}{*}{$\begin{array}{l}\text { Amino acid } \\
\text { residues }\end{array}$} & \multirow[t]{2}{*}{ Sequence } & \multicolumn{2}{|c|}{ Mass } & \multicolumn{2}{|c|}{$\begin{array}{c}\text { Numbers of HNE } \\
\text { adducts }^{b}\end{array}$} \\
\hline & & & & & Theoretical & Observed & Michael & Schiff \\
\hline \multirow[t]{14}{*}{$\alpha$-Spectrin } & $1^{\mathrm{a}}$ & $3-4$ & $257-271$ & QKALSNAANLQRFKR & 1743.99 & 2057.18 & 2 & 0 \\
\hline & 2 & 4 & $295-307$ & DLVASEGLFHSHK & 1438.72 & 1597.85 & 1 & 0 \\
\hline & $3^{\mathrm{a}}$ & 4 & $312-326$ & NLAVMSDKVKELCAK & 1647.87 & 2117.21 & 3 & 0 \\
\hline & 4 & 4 & $322-329$ & ELCAKAEK & 890.45 & 1207.65 & 2 & 0 \\
\hline & $5^{\mathrm{a}}$ & 5 & $412-416$ & HQQHK & 676.34 & 989.56 & 2 & 0 \\
\hline & 6 & 7 & $569-575$ & EKAATRR & 830.47 & 989.56 & 1 & 0 \\
\hline & $8^{\mathrm{a}}$ & 13 & $1225-1230$ & HEGFER & 773.35 & 930.49 & 1 & 0 \\
\hline & $9^{\mathrm{a}}$ & $13-14$ & $1282-1290$ & KESLNEAQK & 1045.54 & 1202.70 & 1 & 0 \\
\hline & 10 & $13-14$ & $1283-1299$ & ESLNEAQKFYLFLSKAR & 2043.08 & 2202.15 & 1 & 0 \\
\hline & $11^{\mathrm{a}}$ & 16 & $1586-1594$ & EHVVDHLLER & 1233.59 & 1546.89 & 2 & 0 \\
\hline & 12 & 17 & $1648-1659$ & KHQLLEREMLAR & 1522.84 & 1663.83 & 0 & 1 \\
\hline & 13 & 18 & $1744-1756$ & DLQGVQNLLKKHK & 1519.88 & 1818.94 & 1 & 1 \\
\hline & $14^{\mathrm{a}}$ & 20 & 2016-2027 & WEQLLEASAVHR & 1437.74 & 1594.95 & 1 & 0 \\
\hline & 15 & 21 & $2133-2141$ & HLSDIIEER & 1110.57 & 1269.71 & 1 & 0 \\
\hline \multirow[t]{8}{*}{$\beta$-Spectrin } & 1 & $\mathrm{Nt}$ & $45-52$ & IKALADER & 914.52 & 1055.56 & 0 & 1 \\
\hline & $2^{a}$ & $\mathrm{Nt}$ & $75-86$ & ITDLYKDLRDGR & 1463.77 & 1620.88 & 1 & 0 \\
\hline & $3^{\mathrm{a}}$ & $\mathrm{Nt}$ & $110-118$ & IHCLENVDK & 1069.52 & 1382.65 & 2 & 0 \\
\hline & $4^{\mathrm{a}}$ & $\mathrm{Nt}$ & $110-124$ & IHCLENVDKALQFLK & 1769.95 & 2239.24 & 3 & 0 \\
\hline & 5 & $\mathrm{Nt}$ & $110-127$ & IHCLENVDKALQFLKEQR & 2183.15 & 2622.46 & 1 & 2 \\
\hline & 6 & $\mathrm{Nt}$ & $227-243$ & DSNARHNLEHAFNVAER & 1978.94 & 2138.08 & 1 & 0 \\
\hline & $7^{\mathrm{a}}$ & 1 & $293-302$ & VIDHAIETEK & 1153.60 & 1310.70 & 1 & 0 \\
\hline & $8^{\mathrm{a}}$ & 2 & $491-498$ & ENYHDQKR & 1088.50 & 1401.74 & 2 & 0 \\
\hline
\end{tabular}




\begin{tabular}{ccrlccc}
9 & 3 & $570-593$ & HKLMEADIAIQGDKVKAITAATLK & 2564.44 & 2705.48 & 0 \\
$10^{\mathrm{a}}$ & 4 & $631-641$ & TQLEQSKRLWK & 1415.79 & 1572.79 & 1 \\
$11^{\mathrm{a}}$ & 7 & $956-975$ & VHTLCVDCEETSKWITDKTK & 2335.12 & 2705.48 & 2 \\
12 & 7 & $974-996$ & TKVVESTKDLGRDLAGIIAIQRK & 2510.45 & 2827.62 & 2 \\
13 & 9 & $1161-1175$ & SHTLAQCLGFQEFQK & 1735.84 & 1894.98 & 1 \\
14 & 11 & $1404-1420$ & SDDPRKDLTSVNRMLAK & 1945.00 & 2104.03 & 1 \\
15 & 11 & $1417-1431$ & MLAKLKRVEDQVNVR & 1798.02 & 1938.91 & 0 \\
16 & 14 & $1671-1684$ & HYAGLKDVAEERKR & 1670.89 & 1830.01 & 1 \\
17 & 14 & $1677-1696$ & DVAEERKRKLENMYHLFQLK & 2546.34 & 2705.48 & 1 \\
$18^{\mathrm{a}}$ & 17 & $1991-2004$ & RKEMNEKWEARWER & 1946.95 & 2260.14 & 2 \\
\hline
\end{tabular}

${ }^{\mathrm{a}}$ Obtained for $\alpha$ - and $\beta$-spectrin without reduction. Other HNE-peptides were detected for polypeptides reduced with $\mathrm{NaBH} 4$ prior to tryptic digestion.

${ }^{\mathrm{b}}$ Numbers of deduced HNE-amino acid adducts in each peptide. 


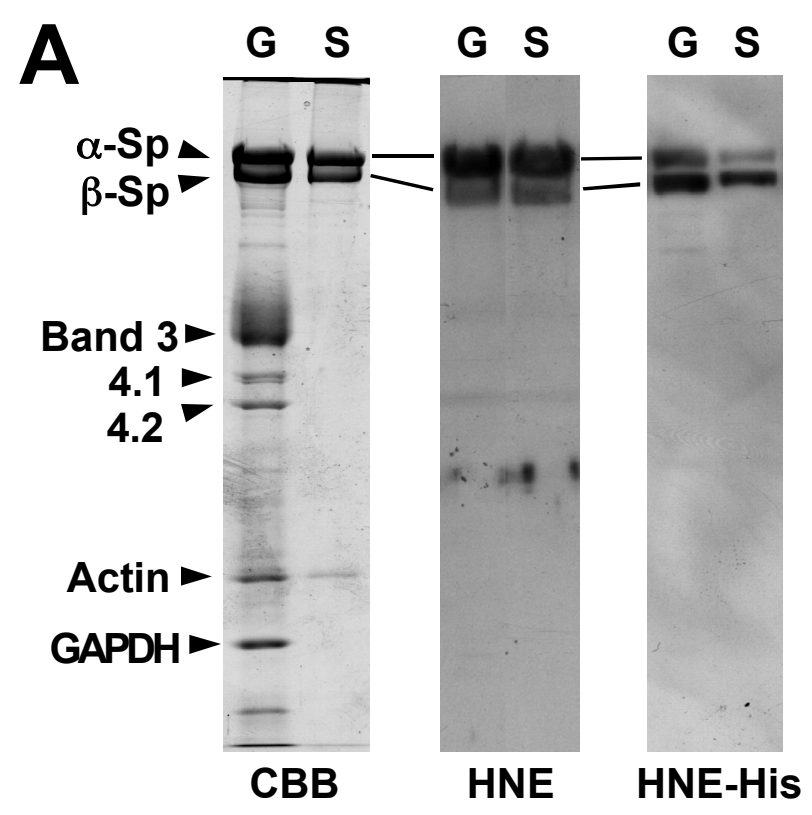

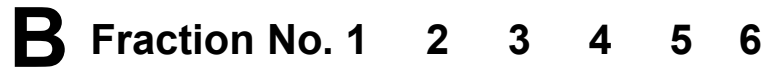
HNE $\begin{aligned} & \alpha-S p \\ & \beta-S p\end{aligned}$

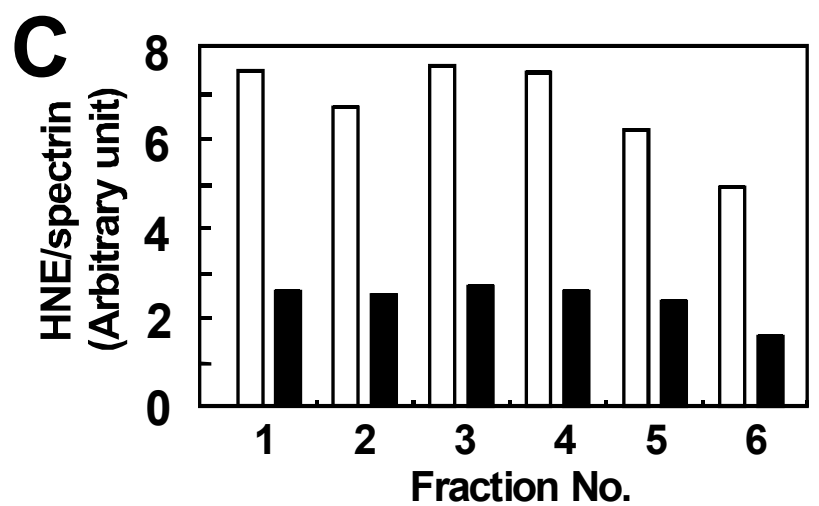

Figure 1 Arashiki et al. 


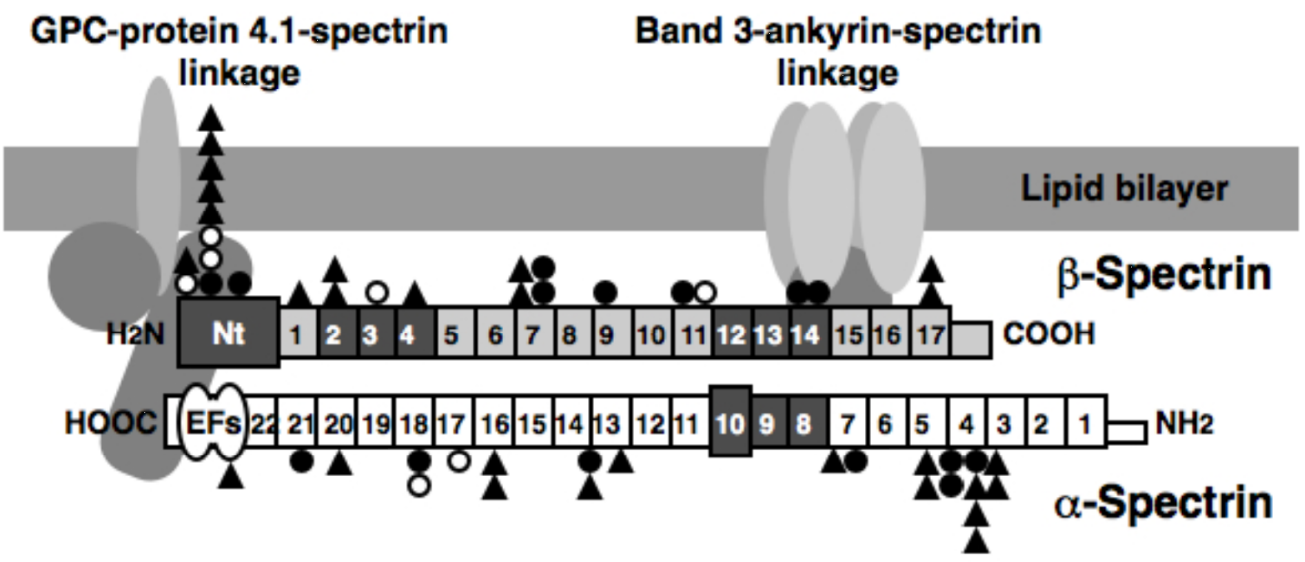

Figure 2 Arashiki et al. 


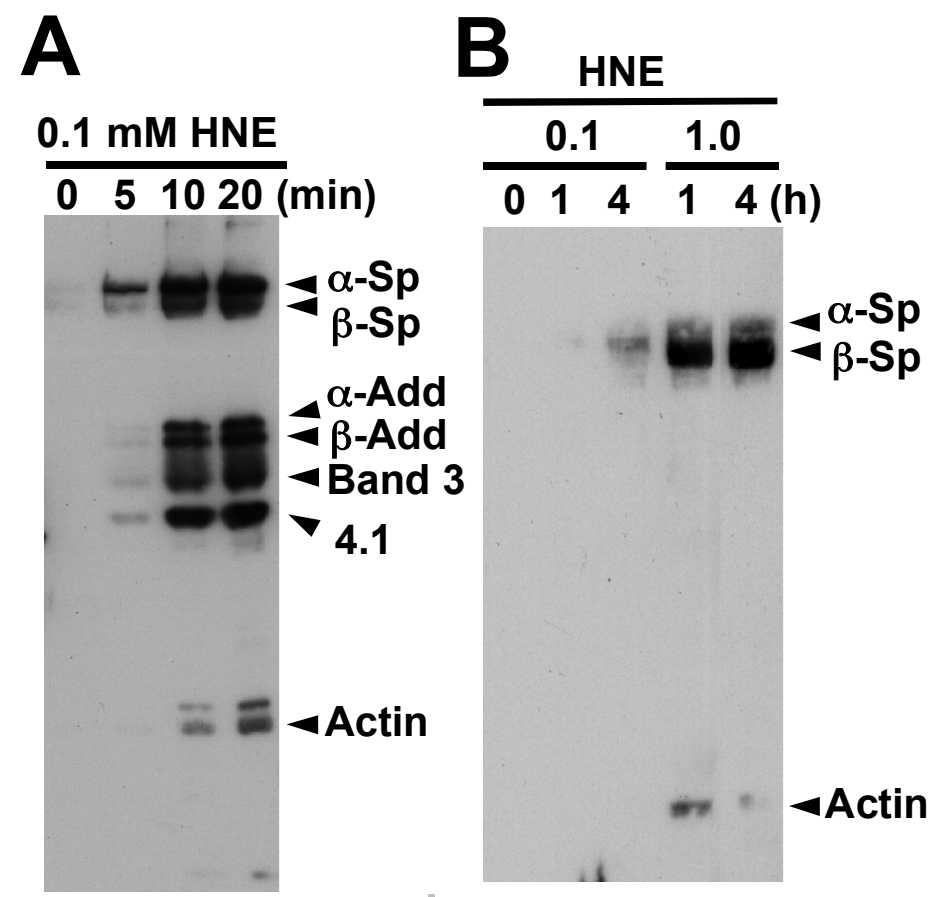

Figure 3 Arashiki et al. 


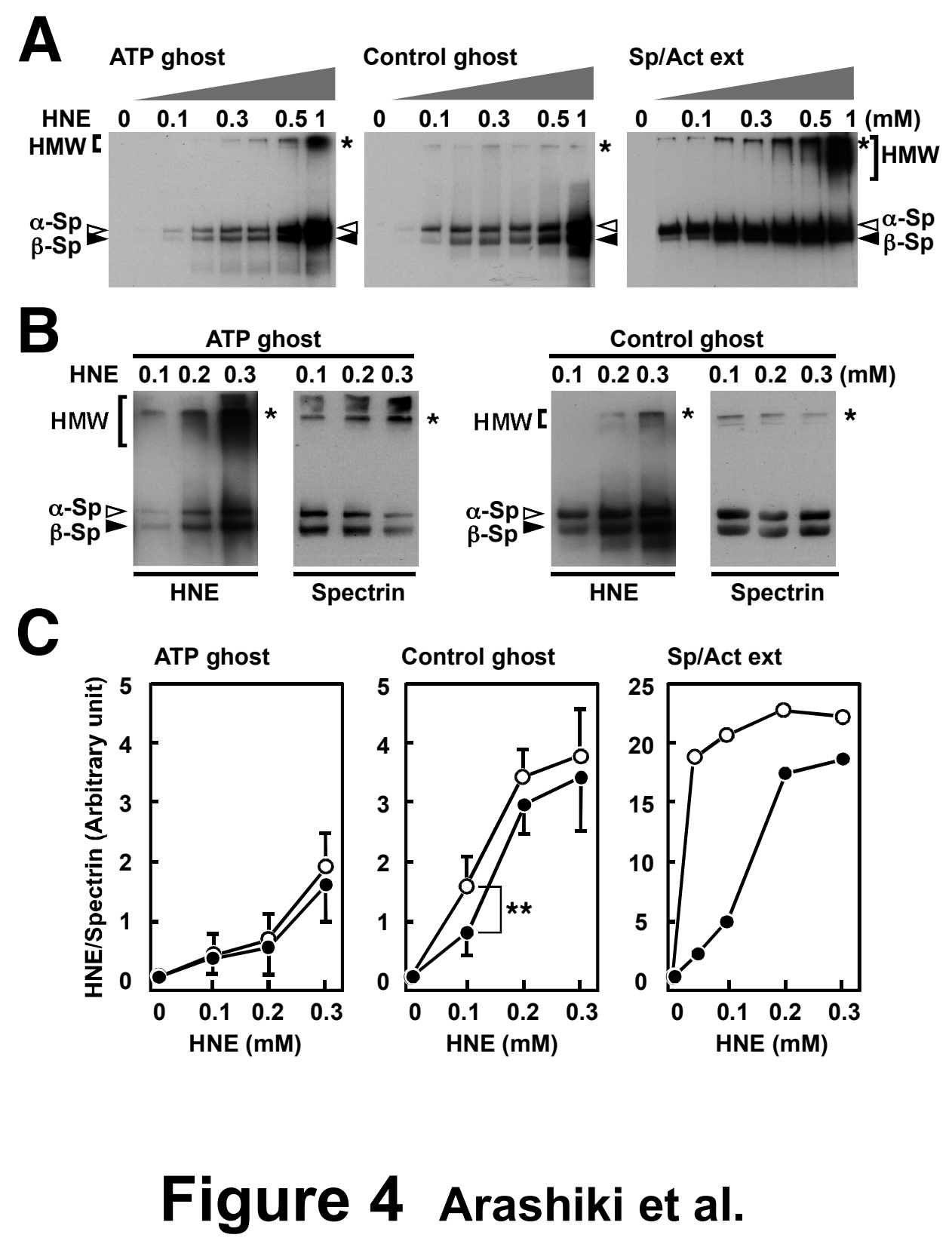

